\section{How to manage asparaginase hypersensitivity in acute lymphoblastic leukemia}

\author{
Michael J Burke*
}

\begin{abstract}
Outcomes for children with acute lymphoblastic leukemia (ALL) have improved significantly in recent decades, primarily due to dose-intensified, multi-agent chemotherapy regimens, of which asparaginase has played a prominent role. Despite this success, hypersensitivity remains a significant problem, often requiring the termination of asparaginase. Failure to complete the entire asparaginase therapy course due to clinical hypersensitivity, subclinical hypersensitivity (i.e., silent inactivation), or other treatmentrelated toxicity is associated with poor ALL outcomes. Thus, it is critical to rapidly identify patients who develop clinical/subclinical hypersensitivity and switch these patients to an alternate asparaginase formulation. This article provides an overview of asparaginase hypersensitivity, identification and management of hypersensitivity and subclinical hypersensitivity, and issues related to switching patients to asparaginase Erwinia chrysanthemi following hypersensitivity reaction.
\end{abstract}

Acute lymphoblastic leukemia (ALL) remains the most common pediatric malignancy in the USA with an estimated 3000 children and adolescents expected to be diagnosed in 2014 [1]. With current multi-agent chemotherapy regimens, long-term outcomes for children with ALL have greatly improved with overall survival rates now greater than $90 \%$, compared with under $30 \%$ in the 1960 s $[2,3]$. These dramatic improvements in survival are due to a number of factors, arguably one of the most influential being the inclusion and refinement of asparaginase therapy into existing multiagent chemotherapeutic regimens. First identified as the anti-lymphoma agent in guinea pig serum in 1961 [4], asparaginase is now a cornerstone of treatment for ALL and has been incorporated into every pediatric ALL regimen, as well as many adult ALL protocols $[4,5]$.

Despite this widespread success, hypersensitivity to asparaginase remains a commonly reported adverse event and often requires termination of asparaginase treatment. Failure to receive the complete course of asparaginase treatment, due to hypersensitivity or other toxicities, has been associated with inferior outcomes in ALL [6]; however, clinical outcomes can be maintained in cases where asparaginase hypersensitivity is identified rapidly and patients are switched to asparaginase Erwinia chrysanthemi to maintain the asparagine depletion [7-9]. There are currently three asparaginases approved in the USA for the treatment of ALL. Native Escherichia coli asparaginase and pegylated (PEG) asparaginase are both derived from the bacteria $E$. coli and have been widely used in firstline treatment as part of a multi-agent chemotherapy regimen in ALL protocols [5,10]. Distribution of the native E. coli asparaginase formulation recently ceased in the USA [11], although it remains available outside the country and is the only available treatment option in some parts of the world [12]. The third preparation, asparaginase Erwinia chrysanthemi derived from the bacteria Erwinia chrysanthemi, shows marginal cross-reactivity with E. coli-derived preparations and is an effective

*Division of Pediatric Oncology, Medical College of Wisconsin, Milwaukee, WI, USA; Tel.: +1 414955 4170; Fax: +1 414955 6543; mmburke@mcw.edu

\section{KEYWORDS}

- acute lymphoblastic leukemia • asparaginase - asparaginase Erwinia chrysanthemi • hypersensitivity • silent inactivation • therapeutic drug monitoring 
treatment option for patients who develop hypersensitivity to native $E$. coli asparaginase or PEG-asparaginase $[13,14]$. However, important pharmacokinetic differences do exist between the three types of asparaginase and these differences, which are discussed in this article, have important implications for dosing, particularly when switching patients between asparaginase products following a hypersensitivity event.

Acute lymphoblastic leukemia cells are largely unable to synthesize asparagine, and therefore must rely on external sources of this amino acid for cell growth and survival. Healthy non-malignant cells can synthesize asparagine de novo through the action of the enzyme asparagine synthetase [15]. Taking advantage of this key metabolic difference, asparaginase catalyzes the deamination of asparagine into its components, aspartic acid and ammonia, and at sufficient activity levels results in complete depletion of serum asparagine. Prolonged cellular depletion of asparagine yields reduced DNA, RNA, and protein synthesis, and will ultimately activate cell death mechanisms in deprived leukemia cells [15]. While it is not yet known how long leukemia cells must be deprived of asparagine to initiate irreversible cell death mechanisms, studies have shown that a consistent and prolonged exposure to asparaginase is strongly associated with improved outcomes [6]. The pharmacologic goal of asparaginase therapy is therefore to maximize a patient's exposure to the enzyme in order to provide uninterrupted depletion of asparagine and maximize the probability of complete leukemia cell death. The frequent occurrence of anti-asparaginase antibodies and subsequent hypersensitivity represent the most serious challenge to achieving this goal in ALL patients receiving asparaginase therapy.

\section{Hypersensitivity to asparaginase}

Asparaginase is a large protein of bacterial origin and therefore carries the potential to elicit an immune response when administered to patients. Immune responses are characterized by the development of anti-asparaginase antibodies in patients. These antibodies bind to the asparaginase molecule, potentially reducing its enzymatic activity, and initiate a number of downstream effects. The formation of IgG and $\operatorname{IgE}$ antibodies have been associated with the development of clinical allergy following asparaginase administration in patients $[16,17]$.
Immune reactions in patients treated with asparaginase fall into two general categories: clinical hypersensitivity and subclinical hypersensitivity (commonly referred to as 'silent inactivation'). Clinical hypersensitivity is characterized as an allergic reaction with signs and symptoms consistent with an immune response to a known antigen. The majority of clinical hypersensitivity reactions to asparaginase are mild and often manifest as a localized rash or pain around the injection site, when administered by intramuscular (im.) injection (Table 1) [7]. Mild-to-moderate reactions (Common Toxicity Criteria for Adverse Events [CTCAE] Grades 1 and 2) are characterized by flushing, fever, chills and dyspnea. Severe reactions (CTCAE Grades 3 and 4) can include bronchospasm and anaphylaxis [17]. Despite classification as a singular discreet event, most occurrences of clinical hypersensitivity manifest with a combination of symptoms and can vary from mild to severe [7]. Although hypersensitivity is the most common treatment-related toxicity, asparaginase therapy is also associated with a number of less prevalent adverse events; including pancreatitis, hyperglycemia, vomiting, nausea, abdominal pain and diarrhea [18].

The incidence of hypersensitivity reaction is variable and dependent on a number of factors. Reported rates of asparaginase hypersensitivity in patients with ALL range from 3 to $45 \%$ [5,7,19-23], often necessitating the discontinuation of asparaginase therapy and negatively impacting outcomes [24]. However, in patients who switch to asparaginase Erwinia chrysanthemi, approved in the treatment of patients who develop hypersensitivity to $E$. coli-derived asparaginase, leukemia outcomes are similar to patients who never develop clinical hypersensitivity [6,7,9].

The occurrence of anti-asparaginase antibodies in patients with clinical hypersensitivity can be a common event and result in reduced asparaginase activity [7,20,22]. Even low-grade hypersensitivity reactions (Grade 1/2) can be associated with the development of anti-asparaginase antibodies and result in a reduction of asparaginase activity, asparagine depletion, and to a lesser extent, glutamine depletion (Table 2) [24,25]. In fact, asparaginase activity levels are inversely correlated with anti-asparaginase antibody levels, with patients showing no detectable enzyme activity following pronounced antibody formation $[20,26]$. Due to this relationship, patients who experience a severe hypersensitivity reaction are 
Table 1. Clinical characteristics of hypersensitivity reactions to asparaginase in ALL patients enrolled on St Jude's Children's Research Hospital front-line protocol Total XIII-HR.

\begin{tabular}{|c|c|c|c|c|}
\hline \multirow[t]{2}{*}{ Characteristic } & \multicolumn{2}{|c|}{$\begin{array}{l}\text { Escherichia coli } \\
\text { asparaginase }\end{array}$} & \multicolumn{2}{|c|}{$\begin{array}{c}\text { Asparaginase Erwinia } \\
\text { chrysanthemi }\end{array}$} \\
\hline & $\begin{array}{l}\text { With other } \\
\text { manifestations }\end{array}$ & Alone & $\begin{array}{l}\text { With other } \\
\text { manifestations }\end{array}$ & Alone \\
\hline \multicolumn{5}{|l|}{ Localized reaction } \\
\hline Pain & 21 & 6 & 2 & 2 \\
\hline Edema & 18 & 3 & 2 & 1 \\
\hline Urticaria & 2 & 1 & 0 & 0 \\
\hline Erythema & 5 & 8 & 1 & 0 \\
\hline Macular rash & 2 & 0 & 0 & 0 \\
\hline Pruritis & 1 & 0 & 0 & 0 \\
\hline \multicolumn{5}{|l|}{ Generalized reaction } \\
\hline Pain & 4 & 1 & 0 & 0 \\
\hline Erythema & 13 & 7 & 1 & 0 \\
\hline Urticaria & 8 & 5 & 2 & 0 \\
\hline Macular rash & 3 & 2 & 1 & 2 \\
\hline Pruritis & 4 & 0 & 0 & 0 \\
\hline Respiratory distress & 7 & 2 & 0 & 0 \\
\hline Fever & 5 & 0 & 0 & 0 \\
\hline Angioedema & 12 & 1 & 1 & 0 \\
\hline Loss of consciousness & 1 & 0 & 0 & 0 \\
\hline Anaphylaxis & 0 & 1 & 0 & 0 \\
\hline \multicolumn{5}{|l|}{ Hypersensitivity reactions } \\
\hline Localized reaction & 34 & & 5 & \\
\hline Generalized reaction & 35 & & 5 & \\
\hline Localized and generalized reaction & 10 & & 0 & \\
\hline
\end{tabular}

likely to exhibit significantly reduced asparaginase activity and higher serum asparagine levels shortly after dose administration [21].

It is important to note that the development of anti-asparaginase antibodies does not always result in overt signs of an allergic reaction. While patients who develop antibodies are more likely to experience a hypersensitivity reaction, a subset of patients will develop anti-asparaginase antibodies without obvious signs of an immune response [7,20,21]. This condition, referred to as subclinical hypersensitivity or silent inactivation, can be associated with reduced asparaginase activity levels and result in poor outcomes [27,28]. Due to the lack of an outward immune response, the prevalence of subclinical hypersensitivity can be difficult to estimate, but reports have identified rates of $8-44 \%[7,8,22,29]$. Identification of subclinical hypersensitivity requires the realtime measurement of either anti-asparaginase antibodies or serum asparaginase activity levels. Panosyan et al. measured both antibody levels and asparaginase activity in 280 patients with high-risk ALL treated on the Children's Cancer Group (CCG)-1961 protocol (Figure 1) [21]. All patients received nine doses of $6000 \mathrm{IU} / \mathrm{m}^{2}$ native $E$. coli asparaginase during induction on a Monday, Wednesday, Friday schedule. Patients were subsequently stratified into treatment arms and received either native $E$. coli asparaginase or PEG-asparaginase at various dose schedules. The largest subset of patients (Group C; $\mathrm{n}=115$ [41\%]) showed both clinical hypersensitivity and antibody formation, while a minority of patients tested negative for anti-asparaginase antibodies but displayed clinical hypersensitivity (Group $\mathrm{B} ; \mathrm{n}=27$ [10\%]), possibly due to subthreshold antibody levels. Patients showing clinical hypersensitivity (regardless of antibody status) were switched to asparaginase Erwinia chrysanthemi and showed outcomes similar to patients who did not develop hypersensitivity to $E$. coli-derived asparaginase. However, a significant number of patients (Group D; n = 81 [29\%]) developed 
Table 2. Half-life of asparaginase after hypersensitivity reaction

\begin{tabular}{|c|c|c|}
\hline \multirow{2}{*}{ Asparaginase } & \multicolumn{2}{|r|}{ Half-life } \\
\hline & $\begin{array}{l}\text { Before } \\
\text { hypersensitivity }\end{array}$ & $\begin{array}{l}\text { After hypersensitivity to } \\
\text { native E. coli asparaginase }\end{array}$ \\
\hline Native Escherichia coli asparaginase & $1.28 \pm 0.35$ days & Undetectable* \\
\hline PEG-asparaginase & $5.73 \pm 3.24$ days & $1.82 \pm 0.3$ days $^{*}$ \\
\hline
\end{tabular}

subclinical hypersensitivity, the formation of anti-asparaginase antibodies with no clinical signs of hypersensitivity. Importantly, patients who developed subclinical hypersensitivity were not switched to asparaginase Erwinia chrysanthemi and had significantly greater relapse rates compared with the other three patient groups (16 vs $5,7.4$, and $2.6 \% ; \mathrm{p}=0.01$ ). These results highlight the potential impact subclinical hypersensitivity can have on ALL outcomes. A number of studies suggest the risk of developing subclinical hypersensitivity in patients with relapsed ALL is even greater than in patients receiving front-line therapy $[26,30,31]$. In summary, subclinical hypersensitivity presents a serious challenge to the pharmacological goal of asparaginase therapy - sustained asparagine depletion - and a number of pediatric studies now implement therapeutic drug monitoring (TDM) via measurement of asparaginase activity as a means of rapidly identifying patients at risk $[8,29]$.

\section{Hypersensitivity risk factors}

Factors that may influence a patient's likelihood of experiencing an immune response include the asparaginase preparation, intensity and consistency of dosing, route of administration, concurrent chemotherapy, time point in therapy, and patient genetics $[17,19,32]$. There are currently three types of asparaginases approved for use in patients with ALL. Native E. coli asparaginase and PEG-asparaginase are both derived from the same bacterial source, E. coli. The third formulation, asparaginase Erwinia chrysanthemi, is derived from an alternate bacterial source, Erwinia chrysanthemi, and thus carries an entirely distinct immunologic profile. The greatest incidence of reaction is seen with the native $E$. coli asparaginase, with $5-60 \%$ of patients showing an immune response $[5,21,23]$. PEG-asparaginase is generally less immunogenic than the native preparation and has been associated with an incidence of hypersensitivity ranging from 3 to $24 \%[6,19,25,33]$. Due to their common origins, native E. coli asparaginase and PEG-asparaginase show a significant amount of immunogenic overlap or cross-reactivity [14]. Patients who develop hypersensitivity to native $E$. coli asparaginase and are switched to PEG-asparaginase show a greater incidence of hypersensitivity to PEG-asparaginase compared with patients who receive PEG-asparaginase treatment at induction [19].

As asparaginase Erwinia chrysanthemi shows little cross-reactivity with the $E$. coli-derived asparaginases, it can be successfully administered to patients with ALL following a prior hypersensitivity reaction to native $E$. coli or PEG-asparaginase $[14,27]$. Thus, patients who would otherwise have to discontinue asparaginase treatment are able to complete their prescribed regimen [34]. As a treatment for ALL patients with a prior history of asparaginase hypersensitivity, asparaginase Erwinia chrysanthem $i$ has been associated with hypersensitivity (Grades 1-4) in 6-33\% of patients [5,19,27,34-37]. However, more severe hypersensitivity reactions (Grades 2/3) were limited to $10.9 \%$ (six out of 55) of patients receiving asparaginase Erwinia chrysanthemi in the pivotal Children's Oncology Group (COG) AALL07P2 study, with no patients experiencing Grade 4 hypersensitivity [13]. In a larger compassionate-use trial, the safety of asparaginase Erwinia chrysanthemi was evaluated in 940 patients with ALL who experienced at least a Grade 2 hypersensitivity reaction to E. coli-derived asparaginase [18]. In this large patient population, $13.6 \%$ (136 out of 940) of patients developed a hypersensitivity reaction to asparaginase Erwinia chrysanthemi of any grade but only 3.6\% (34 out of 940) of patients experienced a Grade 3/4 hypersensitivity reaction. The incidence of other asparaginase-associated adverse events (e.g., pancreatitis, hyperglycemia, vomiting, nausea and abdominal pain) was reportedly below $4 \%$ in this large patient population. 


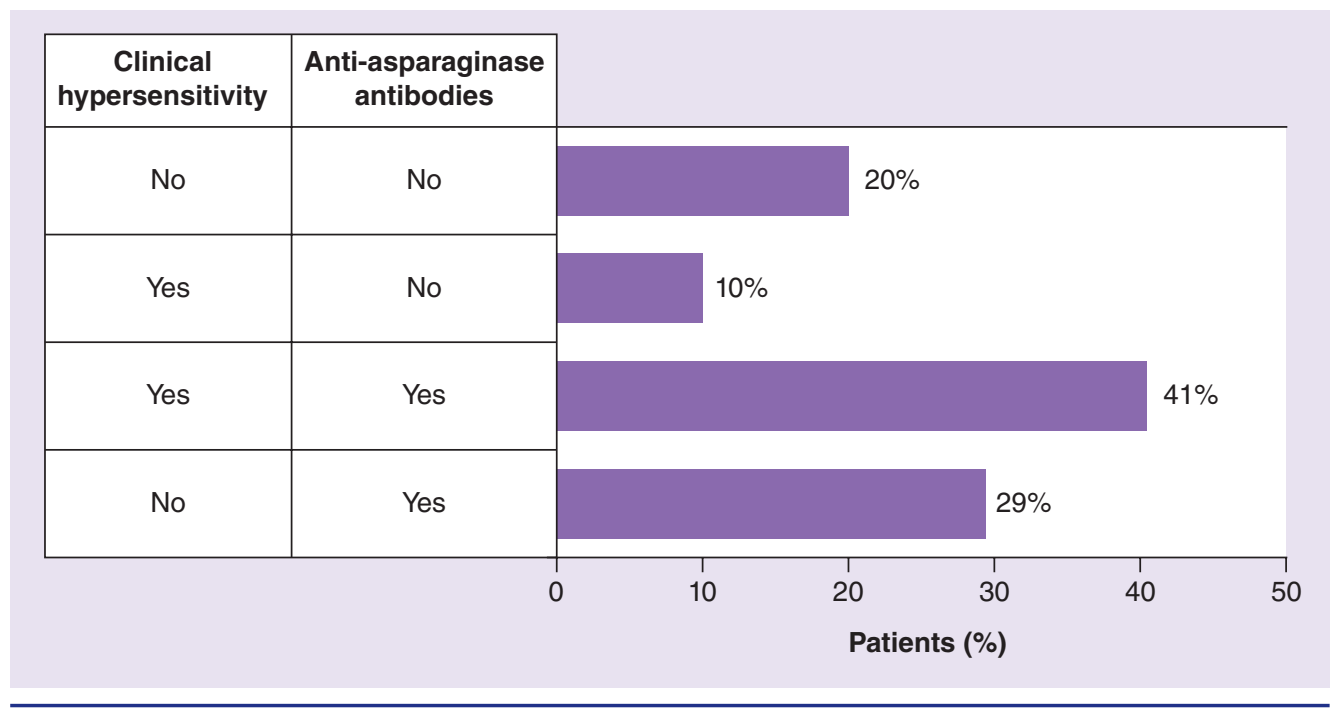

Figure 1. Relationship between clinical hypersensitivity and anti-asparaginase antibodies in CCG-1961.

CCG: Children's Cancer Group.

Adapted with permission from [21].

Corticosteroids are a universal component of ALL treatment protocols and are often used as pre-medication to lower the risk of hypersensitivity to asparaginase treatment, particularly in adult studies [32]. Although no randomized trial directly compares the incidence of hypersensitivity events between patients pre-medicated with corticosteroids and those not given corticosteroids, comparisons of hypersensitivity rates across clinical trials suggest reduced hypersensitivity rates when corticosteroids are used [21,25,38,39]. Patients in the CCG-1961 study were treated with either native E. coli asparaginase or PEGasparaginase following induction and did not receive corticosteroids during the consolidation phase. Patients in this study showed a relatively high incidence of hypersensitivity compared with patients in the CCG-1962 study, in which asparaginase was administered concurrently with corticosteroids [21,25]. However, caution should be raised, as it is currently unknown whether corticosteroid immunosuppression can influence the incidence of subclinical hypersensitivity in patients. Clinical hypersensitivity is often the most direct indicator that a patient has developed anti-asparaginase antibodies and may therefore have reduced asparaginase activity. The use of corticosteroids during asparaginase therapy might mask the occurrence of hypersensitivity and the associated reduction in asparaginase activity. Patients with sufficiently reduced asparaginase activity due to antibody formation should be switched to an alternate asparaginase preparation as soon as clinically possible to assure optimal asparagine depletion. Often this can be done within 48-72 $\mathrm{h}$ after the hypersensitivity event has occurred, as long as all clinical symptoms of the reaction have resolved. Failure to switch patients who have reduced asparaginase activity levels can negatively impact their outcomes [8].

The route of administration of asparaginase can also influence the risk of a hypersensitivity reaction. Earlier studies have shown an increased incidence of anaphylaxis with intravenous (iv.) compared with im. administration of asparaginase [40]. However, recent reports show comparable rates with either mode of administration $[6,7,16,25,41-46]$. Studies that have directly compared the two methods have been conflicting and difficult to interpret. A recent report by August et al. retrospectively evaluated the incidence of hypersensitivity in patients treated with PEGasparaginase between January 2006 and May 2008 at a single center [47]. The overall incidence of hypersensitivity to PEG-asparaginase was $10.3 \%$. Subanalysis by route of drug administration (iv. vs im.) showed comparable incidence of hypersensitivity in patients who received iv. only $(n=16)$ versus im. only $(n=27)$ PEG-asparaginase, $12.5 \%$ and $11.1 \%$, respectively. Additionally, there was no difference between severe hypersensitivity reactions (Grade 3/4) in the two groups, occurring only once after 119 total doses 
of iv. PEG-asparaginase (0.8\%) and once after 215 total doses of im. PEG-asparaginase (0.5\%; $\mathrm{p}=1.0)$. These results contrast with data from Pidaparti and Bostrom, who found a greater incidence of hypersensitivity following iv. administration $(\mathrm{n}=11)$ of PEG-asparaginase compared with im. $(\mathrm{n}=186)$ in children with ALL (36 vs $9 \% ; \mathrm{p}=0.019$ ) [48]. However, in the Pidaparti study, the majority of reactions in the im. group were CTCAE Grade 3-4, compared with only Grade 1-2 in the iv. group. A limitation of both studies was a low sample size in one or more subgroups, making results difficult to generalize to the larger ALL population. Reviews evaluating the relative incidence of hypersensitivity between im. and iv. administration of PEG-asparaginase across different trial populations suggest rates are comparable [19,49]. The pharmacokinetics of asparaginase activity differ greatly between routes of administration with iv. showing a more rapid time-to-peak activity compared with im. injections [50]. This difference may have implications for the timing of hypersensitivity events following drug administration. Petersen et al. showed hypersensitivity reactions were more likely to occur within the 30-min period immediately following iv. administration, compared with im. injections, in which reactions typically occurred much later [51]. The researchers observed that all hypersensitivity reactions (14 out of 14 ) occurred within $30 \mathrm{~min}$ of administration in the iv. patient group, compared with just one out of 11 reactions occurring in the im. group within the 30 -min window ( $\mathrm{p}=0.0001)$. These results suggest that patients may require observation for a shorter duration of time when asparaginase is administered iv., as systemic exposure to the drug is more rapid. Administration via the iv. route also allows healthcare professionals to terminate an infusion, in the case of a severe allergic reaction, thus potentially sparing the patient from full exposure to the drug, an option not available following im. administration.

Hypersensitivity reactions to asparaginase are more common following a prolonged break in asparaginase treatment and occur almost exclusively in post-induction phases of chemotherapy [52]. In a report by Woo et al., 22 patients experienced a hypersensitivity reaction to native $E$. coli asparaginase after a median of 11 doses (during re-induction) [7]. Vrooman and colleagues had similar findings, observing hypersensitivity in 45 patients after a median of five post-induction doses of native $E$. coli asparaginase [27]. Treatment protocols that use a more intensive asparaginase dosing schedule without any 'treatment holidays' or breaks in asparaginase treatment have been associated with reduced immunogenicity [53]. Additionally, more intensive ALL regimens may be associated with reduced immunogenicity given their greater immunosuppressive effects [37,54].

Patient characteristics, such as gender and age, seem to have little impact on the risk of hypersensitivity reaction to asparaginase, although relatively higher levels of anti-asparaginase antibodies have been identified in males $[6,21,35]$. Genetic differences have been shown to influence the likelihood of developing hypersensitivity to asparaginase. Chen et al. evaluated patient demographics and genetic variations that might contribute to the risk of asparaginase allergy in 498 children with newly diagnosed ALL [54]. Of the clinical variables analyzed in this study, including gender, age, racial ancestry and ALL-treatment risk group, the lowest risk of hypersensitivity was among patients of American-Indian ancestry $(p=0.016)$, while patients assigned to the ALL low-risk risk group had the highest risk of hypersensitivity $(\mathrm{p}=0.041)$. Results of a genome-wide analysis revealed genetic variations in GRIA1 (located at 5q33) were associated with an increased incidence of hypersensitivity.

\section{Management of hypersensitivity}

Patients should typically be observed for at least $1 \mathrm{~h}$ following administration of asparaginase to monitor for adverse reactions. Nursing staff should monitor patients for signs of a hypersensitivity reaction and resources for treating anaphylaxis should be readily available. Due to the longer half-life of PEG-asparaginase, hypersensitivity reactions may appear hours after the administration of the drug $[24,55]$. The timing of hypersensitivity reactions depends on the method of delivery and, although much less common, reactions have been reported several hours after administration [51,55]. Patients and caregivers should therefore be instructed to watch for signs and symptoms of a delayed clinical hypersensitivity reaction at home and be given detailed instructions regarding how to handle such an event.

Due to the discontinuation of native $E$. coli asparaginase in the USA, PEG-asparaginase is now used as first-line treatment in multi-agent chemotherapy regimens in all US pediatric 
ALL protocols, as well as in some adult ALL trials [5,11]. Rates of hypersensitivity reactions for patients receiving PEG-asparaginase have been reported at 3-24\%, with anti-asparaginase antibodies found in up to $60 \%$ of these patients $[6,19,25,33]$. Patients who develop at least a Grade 2 hypersensitivity reaction to PEG-asparaginase typically have three treatment options [56]:

- Termination of all asparaginase treatment;

- Attempt to continue treatment with PEGasparaginase through a desensitization protocol or the addition of hypersensitivity prophylaxis using a combination of acetaminophen, diphenhydramine and steroids;

- Switch the patient to asparaginase Erwinia chrysanthemi.

Termination of all subsequent asparaginase therapy is not advised, as reduced duration of asparaginase treatment has been clearly associated with inferior outcomes in ALL [5,6,57]. Desensitization protocols have been described with some success, but are largely utilized in countries with reduced access to viable asparaginase alternatives [12]. Additionally, although continued asparaginase therapy with corticosteroid premedication is possible, it has shown limited effectiveness and may simply mask symptoms of subclinical hypersensitivity. Woo et al. attempted to re-challenge 12 patients who had a prior hypersensitivity reaction to $E$. coli asparaginase by premedicating with diphenhydramine or corticosteroids. The researchers found a striking $83 \%$ of these patients experienced a second hypersensitivity reaction and were subsequently switched to asparaginase Erwinia chrysanthemi [44].

In the event of an allergic reaction to PEGasparaginase (Grade $\geq 2$ ), patients should be switched to asparaginase Erwinia chrysanthemi. Asparaginase Erwinia chrysanthemi has shown limited cross-reactivity with $E$. coli-derived asparaginase formulations and thus allows the majority of patients who experience hypersensitivity to native E. coliasparaginase or PEG-asparaginase to complete their prescribed treatment regimen $[14,56]$. In a study from the Dana Farber Cancer Institute, 42 patients with hypersensitivity to native $E$. coli asparaginase were switched to asparaginase Erwinia chrysanthemi. The majority of these patients (81\%) were able to finish $\geq 26$ weeks of their planned asparaginase therapy and reported a 5.4-year event-free survival (EFS), comparable with patients who never experienced hypersensitivity $(86 \% \pm 5 \%$ vs $81 \% \pm 3 \%$; $\mathrm{p}=0.55)$ [27]. A recent study by Tong et al. found that $97 \%$ of patients switched to asparaginase Erwinia chrysanthemi following hypersensitivity to PEG-asparaginase were able to complete their full course of treatment [29]. Of the 59 patients who switched, only three patients developed signs of a severe clinical hypersensitivity reaction to asparaginase Erwinia chrysanthemi and no patient showed reduced asparaginase activity levels as a result of subclinical hypersensitivity.

Although all three asparaginase formulations share the same mechanism of action, pharmacokinetic properties differ greatly between PEG-asparaginase and asparaginase Erwinia chrysanthemi. These differences have important implications for dosing that must be accounted for when switching between preparations. For example, the half-life for PEG-asparaginase is reported at $5.7 \pm 3.2$ days, compared with $15.6 \pm 3.1 \mathrm{~h}$ for asparaginase Erwinia chrysanthemi [24]. Due to its longer half-life, PEGasparaginase is metabolized at a slower rate and therefore provides a relatively longer period of therapeutic activity and asparagine depletion. With asparaginase Erwinia chrysanthemi, in order to maintain sufficient asparaginase activity and adequate asparagine depletion, patients must receive six doses of asparaginase Erwinia chrysanthemi for each planned dose of PEGasparaginase (3 days a week on a Monday, Wednesday, Friday schedule).

In a recent report from the COG, 55 patients who developed hypersensitivity to PEG-asparaginase were switched to $25,000 \mathrm{IU} / \mathrm{m}^{2}$ asparaginase Erwinia chrysanthemi [13]. To compensate for the shorter half-life, six doses of asparaginase Erwinia chrysanthemi were substituted for each remaining dose of PEG-asparaginase and patients were given im. injections on a Monday, Wednesday, Friday schedule. The goal of this study was to evaluate both 48- and 72-h asparaginase activity levels to determine if the current dose structure resulted in sufficient asparaginase activity to maintain asparagine depletion in the majority of patients (asparaginase level $\geq 0.1 \mathrm{IU} / \mathrm{ml}$ ) [58]. Results showed that asparaginase Erwinia chrysanthemi was well tolerated and, importantly, maintained asparaginase activity $>0.1 \mathrm{IU} / \mathrm{ml}$ at both 48 and $72 \mathrm{~h}$ post-injection in all patients tested. Activity levels were expectedly higher prior to the Wednesday/ Friday injections ( $48 \mathrm{~h}$ post-injection), compared with measurements prior to the Monday injection (72 $\mathrm{h}$ post-injection). However, this dosing 
scheme was deemed to provide sufficient asparaginase activity levels and allowed the drug to be administered during the operating hours of most outpatient clinics. The results of this study provided the basis for the 2011 US FDA approval of Erwinaze $^{\circledR}$ (asparaginase Erwinia chrysanthemi; Jazz Pharmaceuticals, CA, USA) and established the current recommendations for dose-substitution with asparaginase Erwinia chrysanthemi following hypersensitivity reaction to native $E$. coli asparaginase or PEG-asparaginase (Figure 2) [59].

There is less clinical evidence available regarding the optimal time to transition between asparaginase preparations after a hypersensitivity reaction occurs. When clinical hypersensitivity is identified and a patient is to be switched to asparaginase Erwinia chrysanthemi, the treating team has two treatment options:

- Begin treatment with asparaginase Erwinia chrysanthemi at the time of the patient's next scheduled PEG-asparaginase dose;

- Begin treatment with asparaginase Erwinia chrysanthemi as soon as clinically possible, ideally within $48-72 \mathrm{~h}$ following the hypersensitivity reaction.

The rationale supporting the latter is that patients with hypersensitivity are likely to develop anti-asparaginase antibodies and often will have no detectable asparaginase activity shortly following administration of the drug [21]. Because of this, it is likely that patients receive no pharmacological benefit from the hypersensitivity-associated dose; thus, the period between hypersensitivity and initiation of subsequent treatment with asparaginase Erwinia chrysanthemi is comparable to a lapse in treatment.

To substitute for PEG-asparaginase: Asparaginase Erwinia chrysanthemi 25,000 IU/m² im. three-times a week (Monday/Wednesday/ Friday) for six doses for each planned dose of PEG-asparaginase.

To substitute for native Escherichia coli asparaginase:

Asparaginase Erwinia chrysanthemi $25,000 \mathrm{IU} / \mathrm{m}^{2}$ im. for each planned dose of native E. coli asparaginase.

Figure 2. Recommended substitution of asparaginase Erwinia chrysanthemi for Escherichia coli-derived asparaginases. im.: Intramuscular; PEG: Pegylated.

Adapted from [59].
Theoretically, this method of replacement would reduce the duration of treatment time patients experiencing hypersensitivity spent at subtherapeutic asparaginase activity levels. However, one potential concern with this approach is that a small subset of hypersensitivity patients $(\sim \leq 10 \%)$ with no detectable anti-asparaginase antibodies might be exposed to unnecessarily high enzyme activity levels during this transition period. As some toxicities have been linked to higher asparaginase activity levels, these patients might be at a greater risk for adverse events [15].

\section{Therapeutic drug monitoring}

TDM refers to the ability to accurately measure asparagine concentrations or asparaginase activity in patients with ALL as a means of assessing treatment efficacy. The use of TDM could further optimize asparaginase treatment, as greater levels of asparaginase activity have been associated with improved outcomes $[6,57,60,61]$. When gaps in asparaginase treatment occur, due to switching asparaginase preparations following clinical hypersensitivity, a period of reduced asparaginase activity can result. In addition, subclinical hypersensitivity can result in prolonged periods of subtherapeutic asparaginase activity in up to $30 \%$ of patients, which can translate into inferior outcomes [8,21]. With TDM, practitioners can pharmacologically evaluate whether an individual patient needs to switch asparaginase preparations due to subclinical hypersensitivity.

In a recent randomized trial of children with newly diagnosed ALL, patients on a drugmonitoring protocol for individualized dosing of $E$. coli asparaginase showed significantly better 5-year EFS compared with patients on a fixed-dose regimen ( 90 vs $82 \%$, respectively; $\mathrm{p}=0.04)[8]$. Study authors attributed this difference primarily to the identification of silent inactivation and the subsequent adjustment in treatment for patients in the drug-monitoring group. Although both groups showed a similar incidence of subclinical hypersensitivity (drugmonitoring: $10 \%$; non-drug-monitoring: $9 \%$ ), only in the drug-monitoring group were patients switched to an alternate asparaginase preparation after silent activation. In this study, the authors defined silent activation as two consecutive measurements of trough asparaginase activity $<0.1 \mathrm{IU} / \mathrm{ml}$, or a single activity measurement $<0.1 \mathrm{IU} / \mathrm{ml}$ combined with the identification of asparaginase antibodies. To expedite the identification of patients with silent inactivation, 
the study protocol called for the immediate testing for asparaginase antibodies following a $<0.1 \mathrm{IU} / \mathrm{ml}$ asparaginase activity test result. Following the switch to an alternate asparaginase, 17 out of 19 patients with silent inactivation $(90 \%)$ had at least one trough asparaginase activity measurement $\geq 0.1 \mathrm{IU} / \mathrm{ml}$, whereas 18 patients with silent inactivation in the nondrug-monitoring group showed no sign of clinical hypersensitivity and were never switched to an alternate asparaginase preparation. Patients with silent inactivation in the non-drug-monitoring group who never switched asparaginase preparations showed reduced 5-year EFS rates compared with patients with silent inactivation in the drug-monitoring group who did switch asparaginase (76 vs $95 \%$, respectively).

As maintaining adequate asparagine depletion remains the pharmacological goal of asparaginase therapy in ALL, TDM is becoming an increasingly utilized clinical tool to evaluate the effectiveness of asparaginase treatment in realtime. Due to some methodological concerns in measuring asparagine serum levels, asparaginase activity is often more practical to measure and has been shown to strongly correlate with asparagine concentrations [41,62]. Activity levels $\geq 0.01 \mathrm{IU} / \mathrm{ml}$ have been shown to result in adequate asparagine serum depletion and a number of ALL treatment protocols have implemented TDM in some form to identify patients with subclinical hypersensitivity $[5,58]$. Results of recent clinical trials suggest TDM could be expanded further to tailor the dose and treatment schedule of asparaginase therapy to the individual patient $[8,29]$.

The measurement of anti-asparaginase antibodies may also be a useful metric to guide treatment management. A recent retrospective analysis of 329 patients with ALL treated according to ALL-BFM protocols found a significant relationship between antibodies and asparaginase activity [26]. All patients received front-line treatment with native $E$. coli asparaginase and second-line treatment with PEGasparaginase, or asparaginase Erwinia chrysanthemi if an allergic reaction was experienced during PEG-asparaginase treatment. Patients with sufficiently high antibodies to native $E$. coli asparaginase ( $>200 \mathrm{AU} / \mathrm{ml})$ showed a significant reduction in asparaginase activity during second-line treatment with PEG-asparaginase, highlighting the cross reactivity between these two enzymes, while patients with moderate native E. coli antibody levels (6.25-200 AU/ml) still showed asparaginase activity $>0.1 \mathrm{IU} / \mathrm{ml}$. The authors suggest antibody levels could be used to predict which patients might benefit most from switching asparaginase formulations.

\section{Conclusion}

In summary, asparaginase therapy historically has been, and continues to be, a critical component of multi-agent chemotherapy for the treatment of ALL. Intensified asparaginase use is associated with significant improvements in outcomes for both pediatric and adult patients with ALL. Despite its efficacy in leukemia therapy, adverse events such as hypersensitivity reactions continue to provide challenges for continuing asparaginase treatment once a reaction occurs. As asparaginase is a large, foreign immunogenic protein, patients are at increased risk for development of anti-asparaginase antibodies, which can significantly impact treatment efficacy. Having the ability to switch asparaginase formulations from the E. coli-derived PEG-asparaginase to asparaginase Erwinia chrysanthemi in ALL patients with clinical hypersensitivity to PEG-asparaginase, allows for the completion of scheduled asparaginase treatment and has been shown to significantly improve survival. Realtime TDM of asparaginase activity in patients is now available and can be used to identify asparaginase levels considered inadequate for asparagine depletion, as well as identify silent inactivation in patients who may not display clinical hypersensitivity. The ability to individualize asparaginase therapy in ALL patients with TDM, optimizing their asparagine depletion by adjusting the PEG-asparaginase dose or switching patients with silent inactivation to an alternate asparaginase preparation may help improve outcomes in those patients.

\section{Future perspective}

Hypersensitivity to asparaginase remains one of the most common reasons for treatment discontinuation in pediatric and adult patients with ALL. A number of factors promise to change the immunogenic risk associated with asparaginase therapy in the coming years. First, with the discontinuation of native E. coli asparaginase in the USA, PEG-asparaginase is now the only available FDA-approved first-line treatment option for patients with ALL. Previously reported rates of clinical hypersensitivity in patients treated on PEG-asparaginase included groups of patients 
with previous exposure to native E. coli asparaginase. Due to the significant cross-reactivity between these two formulations, previously exposed patients were effectively 'primed' to react to the PEGylated formulation [14]. Newly diagnosed patients now begin treatment with PEGasparaginase and should be at a reduced risk for immune reaction, although some studies report $22-25 \%$ of anti-PEG antibodies in healthy blood donors [63]. Second, TDM provides a pharmacological metric to quantify the likely effectiveness of a patient's current asparaginase therapy and guide treatment adjustments. Recently, a commercially available, $\mathrm{L}$-asparaginase activity assay (Clinical Laboratory Improvement Amendmentscertified) was developed for use by AIBioTech (VA, USA) [64]. This assay is minimally invasive, requiring only $2 \mathrm{ml}$ of whole blood, and yields results in $<2.5 \mathrm{~h}$ [65]. This assay will allow practitioners to monitor asparaginase activity and adjust the asparaginase dose and treatment schedule for their patients. Third, clinical trials evaluating iv. administration of asparaginase Erwinia chrysanthemi as part of multi-agent chemotherapy for treatment of ALL in patients with hypersensitivity to PEG-asparaginase have been completed and await FDA approval. If approved, the ability to deliver iv. asparaginase Erwinia chrysanthemi will offer a more convenient and less painful administration route than im. injection, particularly in older patients where multiple injections are required. Lastly, the development of novel asparaginase formulations promises to expand therapeutic options beyond the two enzymes currently available for use. New bacterial sources have been identified for the asparaginase enzyme. Asparaginase derived from the bacterium Wolinella succinogenes may offer asparaginase formulations with lower activity for glutamine, possibly reducing asparaginase-associated toxicity [66]. In addition, a PEGylated recombinant Erwinia-derived asparaginase (pegcrisantaspase) is now in the early

\section{EXECUTIVE SUMMARY}

\section{Introduction}

- Despite the widespread success of asparaginase as part of multi-agent chemotherapy for the treatment of acute lymphoblastic leukemia (ALL), hypersensitivity to asparaginase remains a commonly reported adverse event.

- Frequent occurrence of anti-asparaginase antibodies and subsequent hypersensitivity often require the termination of asparaginase therapy, and represents a serious challenge to the treatment of ALL.

\section{Hypersensitivity to asparaginase}

- Immune reactions in patients treated with asparaginase fall into two general categories: clinical hypersensitivity and subclinical hypersensitivity ('silent inactivation'). Clinical hypersensitivity is the most common dose-limiting toxicity in asparaginase therapy and often manifests as a localized rash or pain. Subclinical hypersensitivity is characterized as the development of anti-asparaginase antibodies without obvious signs of an immune response.

- Both clinical and subclinical hypersensitivity have been associated with reduced asparaginase activity levels and poor outcomes in ALL, if not immediately addressed.

\section{Hypersensitivity risk factors}

- Risk factors for hypersensitivity include: the asparaginase preparation, intensity and consistency of dosing, route of administration, concurrent chemotherapy, time point in therapy and patient genetics.

\section{Management of hypersensitivity}

- Patients who develop a Grade $\geq 2$ hypersensitivity reaction to PEG-asparaginase should be switched to asparaginase Erwinia chrysanthemi.

- Hypersensitivity is commonly associated with the development of anti-asparaginase antibodies and patients with hypersensitivity often show no detectable asparaginase activity shortly following administration of the drug. Therefore, it is recommended that therapy with asparaginase Erwinia chrysanthemi be initiated within 48-72 $\mathrm{h}$ following a hypersensitivity reaction.

\section{Therapeutic drug monitoring}

- Therapeutic drug monitoring can be an effective tool to rapidly identify patients with subclinical hypersensitivity to asparaginase.

- Recent clinical evidence suggests the use of therapeutic drug monitoring could be expanded to tailor the dose and treatment schedule of asparaginase therapy to the individual patient. 
stages of clinical development. As was found with the native $E$. coli formulation, the addition of a PEG conjugate to Erwinia-derived asparaginase should increase the pharmacokinetic half-life and prolong the duration of asparagine depletion obtained from single-dose administration, compared with the current non-PEGylated version of asparaginase Erwinia chrysanthemi.

\section{Financial \& competing interests disclosure}

MJ Burke serves as a consultant to Jazz Pharmaceuticals (CA, USA) and is a member of their speaker program for Erwinaze $^{\circledR}$ in the treatment of acute lymphoblastic leukemia. The Midwest Athletes Against Childhood Cancer (MACC) Fund and the Medical College of Wisconsin Pediatric
Leukemia/Lymphoma Program supported this work. The author has no other relevant affiliations or financial involvement with any organization or entity with a financial interest in or financial conflict with the subject matter or materials discussed in the manuscript apart from those disclosed.

MJ Burke would like to thank Cory Hussar, PhD, of The Curry Rockefeller Group, LLC, Tarrytown, NY, USA, for providing editorial assistance that was supported by Jazz Pharmaceuticals plc or its subsidiaries.

\section{Open access}

This work is licensed under the Creative Commons Attribution-NonCommercial 3.0 Unported License. To view a copy of this license, visit http://creativecommons.org/ licenses/by-nc-nd/3.0/

\section{References}

Papers of special note have been highlighted as:

•• of considerable interest

1 American Cancer Society. Cancer Facts and Figures 2014 (2014).

www.cancer.orgf

2 Pui CH, Robison LL, Look AT. Acute lymphoblastic leukaemia. Lancet371(9617), 1030-1043 (2008).

3 Hunger SP, Loh ML, Whitlock JA et al. Children's Oncology Group's 2013 blueprint for research: acute lymphoblastic leukemia. Pediatr. Blood Cancer 60(6), 957-963 (2013).

4 Broome JD. Evidence that the L-asparaginase activity of guinea pig serum is responsible for its antilymphoma effects. Nature 191, 1114-1115 (1961).

5 Pieters R, Hunger SP, Boos J et al. $\mathrm{L}$-asparaginase treatment in acute lymphoblastic leukemia: a focus on Erwinia asparaginase. Cancer 117(2), 238-249 (2011).

6 Silverman LB, Gelber RD, Dalton VK et al. Improved outcome for children with acute lymphoblastic leukemia: results of DanaFarber Consortium Protocol 91-01. Blood 97(5), 1211-1218 (2001).

7 Woo MH, Hak LJ, Storm MC et al. Hypersensitivity or development of antibodies to asparaginase does not impact treatment outcome of childhood acute lymphoblastic leukemia. J. Clin. Oncol. 18(7), 1525-1532 (2000).

8 Vrooman LM, Stevenson KE, Supko JG et al. Postinduction dexamethasone and individualized dosing of Escherichia coli L-asparaginase each improve outcome of children and adolescents with newly diagnosed acute lymphoblastic leukemia: results from a randomized study - DanaFarber Cancer Institute ALL Consortium Protocol 00-01. J. Clin. Oncol. 31(9), 1202-1210 (2013).

- Randomized clinical trial of individualized dosing and fixed-dosing treatment regimens in newly diagnosed patients with acute lymphoblastic leukemia (ALL).

9 Larson RA, Fretzin MH, Dodge RK, Schiffer CA. Hypersensitivity reactions to $\mathrm{L}$-asparaginase do not impact on the remission duration of adults with acute lymphoblastic leukemia. Leukemia 12(5), 660-665 (1998).

Graham ML. Pegaspargase: a review of clinical studies. Adv. Drug Deliv. Rev. 55(10), 1293-1302 (2003).

11 Keding RE. Discontinuation of Elspar (asparaginase for injection) 10,000 IU (2012). www.fda.gov

12 Akbayram S, Dogan M, Akgun C, Caksen H, Oner AF. A desensitization protocol in children with L-asparaginase hypersensitivity. J. Pediatr. Hematol. Oncol. 32(5), e187-191 (2010).

13 Salzer WL, Asselin B, Supko JG et al. Erwinia asparaginase achieves therapeutic activity after PEGaspargase allergy: a report from the Children's Oncology Group. Blood 122(4), 507-514 (2013).

-• Results of the pivotal COG AALL07P2 clinical trial of patients treated with asparaginase Erwinia chrysanthemi $25,000 \mathrm{IU} / \mathrm{m}^{2}$ on a Monday, Wednesday, Friday schedule.

14 Zalewska-Szewczyk B, Gach A, Wyka K, Bodalski J, Mlynarski W. The cross-reactivity of anti-asparaginase antibodies against different L-asparaginase preparations. Clin. Exp. Med. 9(2), 113-116 (2009).
15 Muller HJ, Boos J. Use of L-asparaginase in childhood ALL. Crit. Rev. Oncol. Hematol. 28(2), 97-113 (1998).

16 Avramis VI, Avramis EV, Hunter W, Long MC. Immunogenicity of native or pegylated E. coli and Erwinia asparaginases assessed by ELISA and surface plasmon resonance (SPR-biacore) assays of IgG antibodies (Ab) in sera from patients with acute lymphoblastic leukemia (ALL). Anticancer Res. 29(1), 299-302 (2009).

17 Shinnick SE, Browning ML, Koontz SE. Managing hypersensitivity to asparaginase in pediatrics, adolescents, and young adults. J. Pediatr. Oncol. Nurs. 30(2), 63-77 (2013).

18 Plourde PV, Jeha S, Hijiya N et al. Safety profile of asparaginase Erwinia chrysanthemi in a large compassionate-use trial. Pediatr. Blood Cancer 61(7), 1232-1238 (2014).

19 Raetz EA, Salzer WL. Tolerability and efficacy of L-asparaginase therapy in pediatric patients with acute lymphoblastic leukemia. J. Pediatr. Hematol. Oncol. 32(7), 554-563 (2010).

20 Liu C, Kawedia JD, Cheng C et al. Clinical utility and implications of asparaginase antibodies in acute lymphoblastic leukemia. Leukemia 26(11), 2303-2309 (2012).

21 Panosyan EH, Seibel NL, Martin-Aragon S et al. Asparaginase antibody and asparaginase activity in children with higherrisk acute lymphoblastic leukemia: Children's Cancer Group Study CCG-1961. J. Pediatr. Hematol. Oncol. 26(4), 217-226 (2004).

-• Clinical trial highlighting poor outcomes in patients with subclinical hypersensitivity (silent inactivation). 
22 Zalewska-Szewczyk B, Andrzejewski W, Mlynarski W, Jedrychowska-Danska K, Witas H, Bodalski J. The anti-asparagines antibodies correlate with $\mathrm{L}$-asparagines activity and may affect clinical outcome of childhood acute lymphoblastic leukemia. Leuk. Lymphoma 48(5), 931-936 (2007).

23 Muller HJ, Beier R, Loning L et al. Pharmacokinetics of native Escherichia coli asparaginase (Asparaginase medac) and hypersensitivity reactions in ALL-BFM 95 reinduction treatment. Br. J. Haematol. 114(4), 794-799 (2001).

24 Asselin BL, Whitin JC, Coppola DJ, Rupp IP, Sallan SE, Cohen HJ. Comparative pharmacokinetic studies of three asparaginase preparations. J. Clin. Oncol. 11(9), 1780-1786 (1993).

25 Avramis VI, Sencer S, Periclou AP et al. A randomized comparison of native Escherichia coli asparaginase and polyethylene glycol conjugated asparaginase for treatment of children with newly diagnosed standard-risk acute lymphoblastic leukemia: a Children's Cancer Group study. Blood 99(6), 1986-1994 (2002).

26 Willer A, Gerss J, Konig T et al. AntiEscherichia coli asparaginase antibody levels determine the activity of second-line treatment with pegylated E. coli asparaginase: a retrospective analysis within the ALL-BFM trials. Blood 118(22), 5774-5782 (2011).

27 Vrooman LM, Supko JG, Neuberg DS et al. Erwinia asparaginase after allergy to $E$. coli asparaginase in children with acute lymphoblastic leukemia. Pediatr. Blood Cancer 54(2), 199-205 (2010).

28 Asselin BL. The three asparaginases. Comparative pharmacology and optimal use in childhood leukemia. Adv. Exp. Med. Biol. 457, 621-629 (1999).

29 Tong WH, Pieters R, Kaspers GJ et al. A prospective study on drug monitoring of PEGasparaginase and Erwinia asparaginase and asparaginase antibodies in pediatric acute lymphoblastic leukemia. Blood 123(3), 2026-2033 (2014).

30 Kurtzberg J, Asselin B, Bernstein M, Buchanan GR, Pollock BH, Camitta BM. Polyethylene glycol-conjugated L-asparaginase versus native L-asparaginase in combination with standard agents for children with acute lymphoblastic leukemia in second bone marrow relapse: a Children's Oncology Group Study (POG 8866). J. Pediatr. Hematol. Oncol. 33(8), 610-616 (2011).

31 Abshire TC, Pollock BH, Billett AL, Bradley P, Buchanan GR. Weekly polyethylene glycol conjugated L-asparaginase compared with biweekly dosing produces superior induction remission rates in childhood relapsed acute lymphoblastic leukemia: a Pediatric Oncology Group Study. Blood 96(5), 1709-1715 (2000).

32 Stock W, Douer D, Deangelo DJ et al. Prevention and management of asparaginase/ pegasparaginase-associated toxicities in adults and older adolescents: recommendations of an expert panel. Leuk. Lymphoma 52(12), 2237-2253 (2011).

33 Hawkins DS, Park JR, Thomson BG et al. Asparaginase pharmacokinetics after intensive polyethylene glycol-conjugated L-asparaginase therapy for children with relapsed acute lymphoblastic leukemia. Clin. Cancer Res. 10(16), 5335-5341 (2004).

34 Billett AL, Carls A, Gelber RD, Sallan SE. Allergic reactions to Erwinia asparaginase in children with acute lymphoblastic leukemia who had previous allergic reactions to Escherichia coli asparaginase. Cancer 70(1), 201-206 (1992).

35 Moghrabi A, Levy DE, Asselin B et al. Results of the Dana-Farber Cancer Institute ALL Consortium Protocol 95-01 for children with acute lymphoblastic leukemia. Blood 109(3), 896-904 (2007).

36 Albertsen BK, Schroder H, Jakobsen P et al. Antibody formation during intravenous and intramuscular therapy with Erwinia asparaginase. Med. Pediatr. Oncol. 38(5), 310-316 (2002).

37 Albertsen BK, Schroder H, Jakobsen P, Muller HJ, Carlsen NT, Schmiegelow K. Monitoring of Erwinia asparaginase therapy in childhood ALL in the Nordic countries. Br. J. Clin. Pharmacol. 52(4), 433-437 (2001).

38 Albertsen BK, Schroder H, Ingerslev J et al. Comparison of intramuscular therapy with Erwinia asparaginase and asparaginase medac: pharmacokinetics, pharmacodynamics, formation of antibodies and influence on the coagulation system. $\mathrm{Br}$. J. Haematol. 115(4), 983-990 (2001).

39 Wang B, Relling MV, Storm MC et al. Evaluation of immunologic crossreaction of antiasparaginase antibodies in acute lymphoblastic leukemia (ALL) and lymphoma patients. Leukemia 17(8), 1583-1588 (2003).

40 Nesbit M, Chard R, Evans A, Karon M, Hammond GD. Evaluation of intramuscular versus intravenous administration of L-asparaginase in childhood leukemia. Am. J. Pediatr. Hematol. Oncol. 1(1), 9-13 (1979).

41 Avramis VI, Panosyan EH. Pharmacokinetic/pharmacodynamic relationships of asparaginase formulations: the past, the present and recommendations for the future. Clin. Pharmacokinet. 44(4), 367-393 (2005).

42 Panetta JC, Gajjar A, Hijiya N et al. Comparison of native E. coli and PEG asparaginase pharmacokinetics and pharmacodynamics in pediatric acute lymphoblastic leukemia. Clin. Pharmacol. Ther. 86(6), 651-658 (2009).

43 Cheung NK, Chau IY, Coccia PF. Antibody response to Escherichia coli L-asparaginase. Prognostic significance and clinical utility of antibody measurement. Am. J. Pediatr. Hematol. Oncol. 8(2), 99-104 (1986).

44 Woo MH, Hak LJ, Storm MC et al. Antiasparaginase antibodies following $E$. coli asparaginase therapy in pediatric acute lymphoblastic leukemia. Leukemia 12(10), 1527-1533 (1998).

45 Douer D, Yampolsky H, Cohen LJ et al. Pharmacodynamics and safety of intravenous pegaspargase during remission induction in adults aged 55 years or younger with newly diagnosed acute lymphoblastic leukemia. Blood 109(7), 2744-2750 (2007).

46 Aguayo A, Cortes J, Thomas D, Pierce S, Keating M, Kantarjian H. Combination therapy with methotrexate, vincristine, polyethylene-glycol conjugated-asparaginase, and prednisone in the treatment of patients with refractory or recurrent acute lymphoblastic leukemia. Cancer 86(7), 1203-1209 (1999).

47 August KJ, Miller WP, Dalton A, Shinnick S. Comparison of hypersensitivity reactions to PEG-asparaginase in children after intravenous and intramuscular administration. J. Pediatr. Hematol. Oncol. 35(7), e283-286 (2013).

48 Pidaparti M, Bostrom B. Comparison of allergic reactions to pegasparaginase given intravenously versus intramuscularly. Pediatr. Blood Cancer 59(3), 436-439 (2012).

49 Pieters R, Appel I, Kuehnel HJ et al. Pharmacokinetics, pharmacodynamics, efficacy, and safety of a new recombinant asparaginase preparation in children with previously untreated acute lymphoblastic leukemia: a randomized Phase 2 clinical trial. Blood 112(13), 4832-4838 (2008).

50 Albertsen BK, Jakobsen P, Schroder H, Schmiegelow K, Carlsen NT.

Pharmacokinetics of Erwinia asparaginase after intravenous and intramuscular administration. Cancer Chemother. Pharmacol. 48(1), 77-82 (2001).

51 Petersen W, Clark D, Senn S, Cash T, Keller F, Lew G. PEGaspargase administration in children with acute lymphoblastic leukemia. Presented at: The Meeting of the American 
Society of Pediatric Hematology Oncology. Montreal, Canada April 7-10, 2010.

52 Evans WE, Tsiatis A, Rivera G et al. Anaphylactoid reactions to Escherichia coli and Erwinia asparaginase in children with leukemia and lymphoma. Cancer 49(7), 1378-1383 (1982).

53 Asselin BL, Kurtzberg J. Asparaginase In: Asparaginase in Treatment of Acute Leukemias: New Directions for Clinical Research, Pui CH (Ed.). Humana Press, NJ, USA (2003).

54 Chen SH, Pei D, Yang W et al. Genetic variations in GRIA1 on chromosome $5 \mathrm{q} 33$ related to asparaginase hypersensitivity. Clin. Pharmacol. Ther. 88(2), 191-196 (2010).

55 Hunger S, COG Pharmacy Committee. Parental and oral chemotherapy administration guidelines used by the Children's Oncology Group (Version 6). Archives of the Children's Oncology Group (2010).

56 Rizzari C, Conter V, Stary J, Colombini A, Moericke A, Schrappe M. Optimizing asparaginase therapy for acute lymphoblastic leukemia. Curr. Opin. Oncol. 25(Suppl. 1), S1-S9 (2013).

57 Amylon MD, Shuster J, Pullen J et al. Intensive high-dose asparaginase consolidation improves survival for pediatric patients with $\mathrm{T}$ cell acute lymphoblastic leukemia and advanced stage lymphoblastic lymphoma: a Pediatric Oncology Group study. Leukemia 13(3), 335-342 (1999).

58 Riccardi R, Holcenberg JS, Glaubiger DL, Wood JH, Poplack DG. L-asparaginase pharmacokinetics and asparagine levels in cerebrospinal fluid of rhesus monkeys and humans. Cancer Res. 41(11 Pt 1), 4554-4558 (1981).

59 Erwinaze $^{\circledR}[$ Package Insert $]$. Eusa Pharma (USA) Inc., PA, USA, March 2014.

60 Wetzler M, Sanford BL, Kurtzberg J et al. Effective asparagine depletion with pegylated asparaginase results in improved outcomes in adult acute lymphoblastic leukemia: Cancer and Leukemia Group B Study 9511. Blood 109(10), 4164-4167 (2007).
61 Pession A, Valsecchi MG, Masera G et al. Long-term results of a randomized trial on extended use of high dose L-asparaginase for standard risk childhood acute lymphoblastic leukemia. J. Clin. Oncol. 23(28), 7161-7167 (2005).

62 Asselin BL, Lorenson MY, Whitin JC et al. Measurement of serum L-asparagine in the presence of $\mathrm{L}$-asparaginase requires the presence of an $\mathrm{L}$-asparaginase inhibitor. Cancer Res. 51(24), 6568-6573 (1991).

63 Armstrong JK, Leger R, Wenby RB et al. Occurrence of an antibody to poly(ethylene glycol) in normal donors. Blood 102, 556A (2003).

64 Aibiotech Inc. L-asparaginase Assay for Leukemia Patients. December 2013.

65 Aibiotech Inc. Laboratory Instructions Manual Assay of Clinical Samples fo L-Asparaginase Levels. December 2013.

66 Covini D, Tardito S, Bussolati O et al. Expanding targets for a metabolic therapy of cancer: L-asparaginase. Recent Pat. Anticancer Drug Discov. 7(1), 4-13 (2012). 